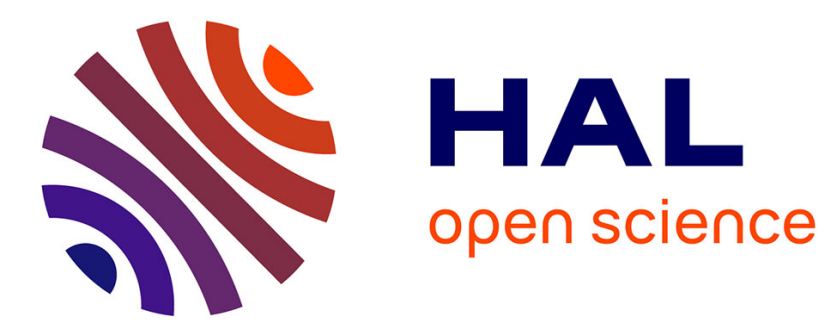

\title{
The Cauchy Problem for Schrödinger-Debye equations
}

Brigitte Bidégaray

\section{To cite this version:}

Brigitte Bidégaray. The Cauchy Problem for Schrödinger-Debye equations. Mathematical Models and Methods in Applied Sciences, 2000, 10 (3), pp.307-315. 10.1142/S0218202500000185 . hal-00319988

\section{HAL Id: hal-00319988 \\ https://hal.science/hal-00319988}

Submitted on 9 Jun 2021

HAL is a multi-disciplinary open access archive for the deposit and dissemination of scientific research documents, whether they are published or not. The documents may come from teaching and research institutions in France or abroad, or from public or private research centers.
L'archive ouverte pluridisciplinaire HAL, est destinée au dépôt et à la diffusion de documents scientifiques de niveau recherche, publiés ou non, émanant des établissements d'enseignement et de recherche français ou étrangers, des laboratoires publics ou privés. 


\title{
The Cauchy Problem for Schrödinger-Debye equations
}

\author{
B. Bidégaray \\ Laboratoire MIP, Université Paul Sabatier \\ 118 route de Narbonne, Toulouse Cedex 4, FRANCE
}

\begin{abstract}
In this article we study the local in time Cauchy problem for the SchrödingerDebye equations which describe the non resonant delayed interaction of an electromagnetic wave with a media. We extend the study to non physical cases such as the three-dimensional case or more general nonlinearities.
\end{abstract}

\section{Introduction.}

We consider Schrödinger-Debye equations

$$
\left\{\begin{array}{l}
i \frac{\partial}{\partial t} A+\frac{c}{2 k n_{0}} \Delta A=\frac{\omega_{0}}{n_{0}} \nu A \\
\tau \frac{\partial}{\partial t} \nu+\nu=n_{2}|A|^{2} .
\end{array}\right.
$$

In these equations $A$ denotes the envelope of a light wave that goes through a media which response is non resonant. However a change $\nu$ is induced in its refraction index (initially $n_{0}$ for an electromagnetic wave of frequency $\omega_{0}$ ) with a slight delay $\tau$. The magnitude (and the sign) of the nonlinear coupling of the matter with the wave is described by the parameter $n_{2}$. The light velocity in the vacuum is denoted by $c$ and $k$ is the wave vector of the incident electromagnetic wave.

In order to simplify proofs we will use the following dimensionless equations keeping however a parameter $\tau$ which for applications has to be thought as small although this fact is of no consequence in the present paper, and replacing $n_{2}$ by $\varepsilon= \pm 1$ to model both focusing and defocusing situations. Results will be exactly the same for the physical system.

$$
\left\{\begin{array}{c}
i \frac{\partial}{\partial t} A+\Delta A=\nu A, \\
\tau \frac{\partial}{\partial t} \nu+\nu=\varepsilon|A|^{2} .
\end{array}\right.
$$


This problem has already been studied in [?, ?]. Here we will enlarge the results obtained in these references. For sake of completeness let us first cite these results, which are valid in the case of a two-dimensional space:

Theorem 1 i) For all $\left(A_{0}, \nu_{0}\right)$ belonging to $H^{s} \times H^{s}$ with $s>1$, equation (??) for the initial data $A(0)=A_{0}, \quad \nu(0)=\nu_{0}$, has a unique solution in $X=L^{\infty}\left(0, T ; H^{s}\right)$ for a small enough $T$ and solutions depend continuously on the initial data.

ii) For all $\left(A_{0}, \nu_{0}\right)$ belonging to $H^{1} \times H^{1}$, equation (??) has a unique solution in $X^{\prime}=L^{\infty}\left(0, T ; H^{1}\right)$ for a small enough $T$.

iii) For all $\left(A_{0}, \nu_{0}\right)$ belonging to $L^{2} \times L^{2}$, equation (??) has a unique solution in $X^{\prime \prime}=L^{4}\left(0, T ; L^{4}\right) \cap \mathcal{C}\left([0, T] ; L^{2}\right)$ for a small enough $T$.

These results have been obtained thanks to a fixed point procedure applied on a Duhamel formulation. To obtain this Duhamel formulation the system (??) has first been written as one integro-differential equation using the fact that

$$
\nu(t)=e^{-t / \tau} \nu_{0}+\frac{\varepsilon}{\tau} \int_{0}^{t} e^{-(t-s) / \tau}|A(s)|^{2} d s .
$$

Hence (??) may read

$$
i \frac{\partial}{\partial t} A+\Delta A=\left(e^{-t / \tau} \nu_{0}+\frac{\varepsilon}{\tau} \int_{0}^{t} e^{-(t-s) / \tau}|A(s)|^{2} d s\right) A
$$

and the fixed point procedure is performed on

$$
A(t)=S(t) A_{0}-i \int_{0}^{t} S(t-s)\left(e^{-s / \tau} \nu_{0}+\frac{\varepsilon}{\tau} \int_{0}^{s} e^{-(s-\theta) / \tau}|A(\theta)|^{2} d \theta\right) A(s) d s
$$

where $S$ is the semi-group associated to the linear Schrödinger equation

$$
i \frac{\partial}{\partial t} A+\Delta A=0
$$

The integro-differential formulation is optional in the case of smooth solutions. In this case the fixed point procedure could as well have been performed on both $A$ and $\nu$. This formulation becomes necessary as soon as weaker solutions are concerned.

Let us first give the equivalent of Theorem ?? in dimensions $N=1,2,3$.

Theorem 2 i) For all $\left(A_{0}, \nu_{0}\right)$ belonging to $H^{s} \times H^{s}$ with $s>N / 2$, equation (??) for the initial data $A(0)=A_{0}, \quad \nu(0)=\nu_{0}$, has a unique solution in $X=L^{\infty}\left(0, T ; H^{s}\right)$ for a small enough $T$ and solutions depend continuously on the initial data.

ii) For all $\left(A_{0}, \nu_{0}\right)$ belonging to $H^{1} \times H^{1}$, equation (??) has a unique solution in $X^{\prime}=L^{\infty}\left(0, T ; H^{1}\right)$ for a small enough $T$.

iii) For all $\left(A_{0}, \nu_{0}\right)$ belonging to $L^{2} \times L^{2}$, equation (??) has a unique solution in $X^{\prime \prime}=L^{8 / N}\left(0, T ; L^{4}\right) \cap \mathcal{C}\left([0, T] ; L^{2}\right)$ for a small enough $T$. 
Proofs are so similar to the two-dimensional case that we do not give them here but refer to [?].

Although the fixed point procedure is performed on variable $A$ only, equation (??) leads to $\nu(T) \in H^{1}$ in case ii) and $\nu(T) \in L^{2}$ in case iii). Nevertheless, unlike the cubic nonlinear Schrödinger equation, there is no conservation law (other than the $L^{2}$ norm of $A$ ) which enables us to transform these local in time results into global ones in the one-dimensional case, for example. Numerical experiments are in progress to give us an idea of how the long-time behavior may look like. These experiments are of course non performed on the integro-differential form but on the coupled equations.

\section{An other integral formulation.}

We use here a trick already applied to the full nonlinear wave equation or the Zakharov equations (see [?, ?]). It consists in setting $F=\frac{\partial A}{\partial t}$ and in writing the equation on $F$ through a formal differentiation of the equation for $A$. We therefore obtain

$$
i \frac{\partial}{\partial t} F+\Delta F=\frac{\partial}{\partial t} \nu A+\nu F
$$

In equation (??) we will replace $\frac{\partial \nu}{\partial t}$ by $-\frac{\nu}{\tau}+\frac{\varepsilon|A|^{2}}{\tau}$ and then replace $A$ by $A_{0}+$ $\int_{0}^{t} F(s) d s$. Thus we obtain the Duhamel formulation

$$
\begin{aligned}
& F(t)=N_{1}[F, \nu] \equiv S(t) F_{0}-i \int_{0}^{t} S(t-s)\left\{\left(-\frac{\nu}{\tau}+\frac{\varepsilon|A(s)|^{2}}{\tau}\right)\right. \\
& \left.\times\left(A_{0}+\int_{0}^{s} F(\theta) d \theta\right)+\nu F\right\} d s, \\
& \nu(t)=N_{2}[F, \nu] \equiv e^{-t / \tau} \nu_{0}+\frac{\varepsilon}{\tau} \int_{0}^{t} e^{-(t-s) / \tau}|A(s)|^{2} d s, \\
& A(t)=(-\Delta+1)^{-1}\left\{i F-\nu\left(A_{0}+\int_{0}^{t} F(s) d s\right)+\left(A_{0}+\int_{0}^{t} F(s) d s\right)\right\} .
\end{aligned}
$$

Since we formally have $i F+\Delta A=\nu A$, we set at time $t=0, i F_{0}=\nu_{0} A_{0}-\Delta A_{0}$. This formulation is only derived formally, we will a posteriori prove that solutions to this system yield solutions to (??).

We shall perform a fixed point procedure for $N=\left(N_{1}, N_{2}\right)$ on the following functional space:

$$
X=\left[L^{\infty}\left(I ; L^{2}\right) \cap L^{8 / N}\left(I ; L^{4}\right)\right] \oplus\left[L^{\infty}\left(I ; L^{4}\right)\right]
$$

Using the same sort of estimates as in [?], we obtain:

$$
\|A\|_{L^{\infty}\left(I ; H^{2}\right)} \leq C\|F\|_{L^{\infty}\left(I ; L^{2}\right)}+C\|\nu\|_{L^{\infty}\left(I ; L^{4}\right)}\left\|A_{0}\right\|_{L^{4}}
$$




$$
\begin{aligned}
& +C T^{1-N / 8}\|\nu\|_{L^{\infty}\left(I ; L^{4}\right)}\|F\|_{L^{8 / N}\left(I ; L^{4}\right)}+\left\|A_{0}\right\|_{L^{2}}+T\|F\|_{L^{\infty}\left(I ; L^{2}\right)}, \\
& \left\|N_{1}(t)\right\|_{L^{4}} \leq\left\|S(t) F_{0}\right\|_{L^{4}}+\int_{0}^{t} C|t-s|^{-N / 4}\left\{\frac{1}{\tau}\|\nu\|_{L^{4}}\left(\left\|A_{0}\right\|_{L^{2}}+\int_{0}^{s}\|F(\theta)\|_{L^{2}} d \theta\right)\right. \\
& \left.+\frac{1}{\tau}\left\||A(s)|^{2}\right\|_{L^{2}}\left(\left\|A_{0}\right\|_{L^{4}}+\int_{0}^{s}\|F(\theta)\|_{L^{4}} d \theta\right)+\|\nu\|_{L^{4}}\|F\|_{L^{2}}\right\} d s, \\
& \left\|N_{1}\right\|_{L^{8 / N}\left(I ; L^{4}\right)} \leq \delta\left\|F_{0}\right\|_{L^{2}}+C \frac{T^{1-N / 8}}{\tau}\|\nu\|_{L^{\infty}\left(I ; L^{4}\right)}\left\|A_{0}\right\|_{L^{2}} \\
& +C \frac{T^{2-N / 8}}{\tau}\|\nu\|_{L^{\infty}\left(I ; L^{4}\right)}\|F\|_{L^{\infty}\left(I ; L^{2}\right)} \\
& +C \frac{T^{1-N / 8}}{\tau}\left\||A|^{2}\right\|_{L^{\infty}\left(I ; L^{2}\right)}\left\|A_{0}\right\|_{L^{4}} \\
& +C \frac{T^{2-N / 4}}{\tau}\left\||A|^{2}\right\|_{L^{\infty}\left(I ; L^{2}\right)}\|F\|_{L^{8 / N}\left(I ; L^{4}\right)} \\
& +C T^{1-N / 8}\|\nu\|_{L^{\infty}\left(I ; L^{4}\right)}\|F\|_{L^{\infty}\left(I ; L^{2}\right)}, \\
& \left\|N_{1}\right\|_{L^{\infty}\left(I ; L^{2}\right)} \leq\left\|F_{0}\right\|_{L^{2}}+C\left\|\frac{1}{\tau}\right\| \nu \|_{L^{4}}\left(\left\|A_{0}\right\|_{L^{2}}+\int_{0}^{s}\|F(\theta)\|_{L^{2}} d \theta\right) \\
& +\frac{1}{\tau}\left\||A(s)|^{2}\right\|_{L^{2}}\left(\left\|A_{0}\right\|_{L^{4}}+\int_{0}^{s}\|F(\theta)\|_{L^{4}} d \theta\right) \\
& +\|\nu\|_{L^{4}}\|F\|_{L^{2}} \|_{L^{8 /(8-N)}(I)} \\
& \leq\left\|F_{0}\right\|_{L^{2}}+C \frac{T^{1-N / 8}}{\tau}\|\nu\|_{L^{\infty}\left(I ; L^{4}\right)}\left\|A_{0}\right\|_{L^{2}} \\
& +C \frac{T^{2-N / 8}}{\tau}\|\nu\|_{L^{\infty}\left(I ; L^{4}\right)}\|F\|_{L^{\infty}\left(I ; L^{2}\right)} \\
& +C \frac{T^{1-N / 8}}{\tau}\left\||A|^{2}\right\|_{L^{\infty}\left(I ; L^{2}\right)}\left\|A_{0}\right\|_{L^{4}} \\
& +C \frac{T^{2-N / 8}}{\tau}\left\||A|^{2}\right\|_{L^{\infty}\left(I ; L^{2}\right)}\|F\|_{L^{8 / N}\left(I ; L^{4}\right)} \\
& +C T^{1-N / 8}\|\nu\|_{L^{\infty}\left(I ; L^{4}\right)}\|F\|_{L^{\infty}\left(I ; L^{2}\right)}, \\
& \left\|N_{2}\right\|_{L^{\infty}\left(I ; L^{4}\right)} \leq\left\|\nu_{0}\right\|_{L^{4}}+\frac{T}{\tau}\left\||A|^{2}\right\|_{L^{\infty}\left(I ; L^{4}\right)} .
\end{aligned}
$$

Unlike estimates obtained in [?], these estimates are not uniform with respect to $\tau$, but this is not our goal here. To obtain a uniform estimate with respect to $\tau$ for $\left\|N_{2}\right\|_{L^{\infty}\left(I ; L^{4}\right)}$ would prevent us to get a $T$ in the estimate and to finish the proof, which ends as follows:

Let us suppose that $\left(A_{0}, \nu_{0}\right) \in H^{2} \times L^{4}$, and let us set

$$
a=\max \left\{\left\|A_{0}\right\|_{L^{2}},\left\|A_{0}\right\|_{L^{4}},\left\|\nu_{0}\right\|_{L^{4}},\left\|\nu_{0} A_{0}-\Delta A_{0}\right\|_{L^{2}}\right\},
$$


which is finite. Let $Y$ be the subset of $X$ defined by

$$
Y=\left\{(F, \nu) \in X /\|F\|_{L^{\infty}\left(I ; L^{2}\right)} \leq 2 a,\|F\|_{L^{8 / N}\left(I ; L^{4}\right)} \leq 2 \delta a,\|\nu\|_{L^{\infty}\left(I ; L^{4}\right)} \leq 2 a\right\} .
$$

The above estimates show that $N$ maps $Y$ into $Y$. Indeed,

$$
\begin{aligned}
\|A\|_{L^{\infty}\left(I ; H^{2}\right)} \leq & 2 C\left(a+a^{2}\right)+4 C T^{1-N / 8} \delta a^{2}+a+2 T a \\
\left\|N_{1}\right\|_{L^{8 / N}\left(I ; L^{4}\right)} \leq & \delta a+2 C \frac{T^{1-N / 8}}{\tau} a^{2}+4 C \frac{T^{2-N / 8}}{\tau} a^{2}+C \frac{T^{1-N / 8}}{\tau}\|A\|_{L^{\infty}\left(I ; H^{2}\right)}^{2} a \\
& +2 C \frac{T^{2-N / 4}}{\tau}\|A\|_{L^{\infty}\left(I ; H^{2}\right)}^{2} \delta a+4 C T^{1-N / 8} a^{2} \\
\left\|N_{1}\right\|_{L^{\infty}\left(I ; L^{2}\right)} \leq & a+2 C \frac{T^{1-N / 8}}{\tau} a^{2}+4 C \frac{T^{2-N / 8}}{\tau} a^{2}+C \frac{T^{1-N / 8}}{\tau}\|A\|_{L^{\infty}\left(I ; H^{2}\right)}^{2} a \\
+ & 2 C \frac{T^{2-N / 8}}{\tau}\|A\|_{L^{\infty}\left(I ; H^{2}\right)}^{2} \delta a+4 C T^{1-N / 8} a^{2}, \\
\left\|N_{2}\right\|_{L^{\infty}\left(I ; L^{4}\right)} \leq & a+\frac{T}{\tau}\|A\|_{L^{\infty}\left(I ; H^{2}\right)}^{2} .
\end{aligned}
$$

For some small enough $T, N[F, \nu] \in Y$.

The same sort of estimates applied on $N[F, \nu]-[F, \nu]$, where both $(F, \nu)$ and $\left(F^{\prime}, \nu^{\prime}\right)$ are solution to (??)-(??)-(??) with the same initial data lead us to show that $N$ is a contraction from $Y$ into $Y$. Hence $N$ has a unique fixed point in $Y$, and

$$
\begin{aligned}
F(t) & =S(t) F_{0}-i \int_{0}^{t} S(t-s)\left\{\left(-\frac{\nu}{\tau}+\frac{\varepsilon|A(s)|^{2}}{\tau}\right)\left(A_{0}+\int_{0}^{s} F(\theta) d \theta\right)+\nu F\right\} d s, \\
\nu(t) & =e^{-t / \tau} \nu_{0}+\frac{\varepsilon}{\tau} \int_{0}^{t} e^{-(t-s) / \tau}|A(s)|^{2} d s, \\
A(t) & =(-\Delta+1)^{-1}\left\{i F-\nu\left(A_{0}+\int_{0}^{t} F(s) d s\right)+\left(A_{0}+\int_{0}^{t} F(s) d s\right)\right\} .
\end{aligned}
$$

Setting $t=0$ in the above equations leads immediately to $F(0)=F_{0}, \nu(0)=\nu_{0}$, $A(0)=A_{0}$.

For more convenience in the following computations we set

$$
B(s)=\left(-\frac{\nu}{\tau}+\frac{\varepsilon|A(s)|^{2}}{\tau}\right)\left(A_{0}+\int_{0}^{s} F(\theta) d \theta\right)+\nu F .
$$

For the results used on the regularization property of operator $S(t)$, the reader may refer to [?]. Since $F_{0} \in L^{2}, S(t) F_{0} \in \mathcal{C}\left(I ; L^{2}\right) \cap \mathcal{C}^{1}\left(I ; H^{-2}\right)$ and $\partial_{t} S(t) F_{0}=$ $i S(t) \Delta F_{0}=i \Delta\left(S(t) F_{0}\right)$. Moreover $B \in L^{\infty}\left(I ; L^{4 / 3}\right) \hookrightarrow L^{1}\left(I ; H^{-1}\right)$ hence

$$
\int_{0}^{t} U(t-s) B(s) d s \in \mathcal{C}\left(I ; H^{-1}\right) \cap W^{1,1}\left(I ; H^{-3}\right) .
$$

Since

$$
\partial_{t} \int_{0}^{t} U(t-s) B(s) d s=i \Delta \int_{0}^{t} U(t-s) B(s) d s+B(t),
$$




$$
\begin{aligned}
\partial_{t} F(t) & =i \Delta\left(U(t) F_{0}+\int_{0}^{t} U(t-s) B(s) d s\right)+i B(t) \\
& =i \Delta F(t)+i B(t)
\end{aligned}
$$

i.e. $i \partial_{t} F(t)+\Delta F(t)=-B(t)$ which is the differential form of (??) and is valid in $\mathcal{C}\left(I ; H^{-1}\right)$.

Since $\nu_{0} \in L^{4}$ and $|A|^{2} \in L^{\infty}\left(I ; L^{4}\right), \nu \in \mathcal{C}\left(I ; L^{4}\right) \cap W^{1, \infty}\left(I ; L^{4}\right)$ and moreover

$$
\begin{aligned}
\partial_{t} \nu & =-\frac{1}{\tau} e^{-t / \tau} \nu_{0}+\frac{\varepsilon}{\tau} \int_{0}^{t}-\frac{1}{\tau} e^{-(t-s) / \tau}|A(s)|^{2} d s+\varepsilon|A(t)|^{2} \\
& =-\frac{\nu}{\tau}+\frac{\varepsilon}{\tau}|A|^{2}
\end{aligned}
$$

This means that $\tau \partial_{t} \nu+\nu=\varepsilon|A|^{2}$, which is the differential form of (??), is valid in $\mathcal{C}\left(I ; L^{4}\right)$.

Moreover we know that $B$ is indeed in $L^{8 /(8-N)}\left(I ; L^{4,3}\right)$, which enables us to conclude that $\int_{0}^{t} S(t-s) B(s) d s \in \mathcal{C}\left(I ; L^{2}\right)$ and therefore $F(t) \in \mathcal{C}\left(I ; L^{2}\right)$. All these results lead to $A \in \mathcal{C}\left(0, T ; H^{2}\right)$. Differentiating equation (??) with respect to time we obtain:

$$
(-\Delta+1) \frac{\partial A}{\partial t}=\left\{i \frac{\partial F}{\partial t}-\frac{\partial \nu}{\partial t}\left(A_{0}+\int_{0}^{t} F(s) d s\right)+\nu F+F\right\} .
$$

which is a priori valid in $\mathcal{C}\left(I ; H^{-1}\right)$. Together with the differential equation on $F$, this gives that $\frac{\partial A}{\partial t}=F$ in $\mathcal{C}\left(I ; H^{-2}\right)$. Furthermore

$$
\frac{\partial A}{\partial t}=(-\Delta+1)^{-1}\left\{i \frac{\partial F}{\partial t}-\frac{\partial \nu}{\partial t}\left(A_{0}+\int_{0}^{t} F(s) d s\right)+\nu F+F\right\}
$$

and $A \in \mathcal{C}^{1}\left(I ; L^{2}\right)$. Equation (??) together with the fact that $\int_{0}^{t} F(s) d s=A(t)-A_{0}$, give

$$
i \frac{\partial}{\partial t} A+\Delta A=\nu A
$$

which is valid in $\mathcal{C}\left(I ; L^{2}\right)$.

Hence this formulation has enabled us to prove the following theorem:

Theorem 3 For all $\left(A_{0}, \nu_{0}\right)$ belonging to $H^{2} \times L^{4}$, equation (??) for the initial data $A(0)=A_{0}, \quad \nu(0)=\nu_{0}$, has a unique solution in $X=\mathcal{C}\left(0, T ; H^{2}\right) \times \mathcal{C}\left(0, T ; L^{4}\right)$ for a small enough $T$ and solutions depend continuously on the initial data.

We may compare this result with the above results. Here we need some strong regularity on $A$ and week regularity on $\nu$. This theorem may also be considered as intermediate between the regular and the weak cases of Theorem ??. The $L^{4}$ regularity for $\nu$ is however very different from the $H^{1}$ or $L^{2}$ regularities asked for in Theorem ??, ii) and iii). The fact that we recover some continuity with respect 
to time for $\nu$ will also be important in some applications (see [?]) as the proof of convergence for numerical schemes.

In the case of the Zakharov system, this huge difference between the regularity for both variables is needed. Here the $H^{2}$-regularity for $A$ is mostly needed because $H^{2}$ is an algebra for all dimensions. $H^{1}$ in dimension 1 and $H^{s}$ in dimension 2 and 3 with $2 \geq s>N / 2$ would have been sufficient. It is however impossible to recover more than a $H^{2}$-regularity on $A$. We may think of higher regularities, using the same approach as above and introducing the new variable $G=\partial_{t}^{2} A$.

Here estimates are not uniform with respect to $\tau$ as we noticed formerly. One drawback is the fact that we are not able to perform a limit as this parameter tends to 0 .

\section{A more general case.}

Going a little further from applications, we may think of studying the following system

$$
\left\{\begin{array}{l}
i \frac{\partial}{\partial t} A+\Delta A=\nu A, \\
\tau \frac{\partial}{\partial t} \nu+\nu=f\left(x,|A|^{2}\right),
\end{array}\right.
$$

where $|f(x, u)| \leq C\left(1+|u|^{\sigma}\right)$ and $\sigma>0$. This might not be so far from actual models for optics since Kerr media are not the only ones to be studied. Nevertheless we have never seen the model (??) in the literature. Of course it is inspired from the general nonlinear Schrödinger equations and results are as well easy to find as soon as we deal with algebras. Otherwise we have to consider the notion of critical value for the nonlinearity. This is the maximal value of $\sigma$ for which one may hope to find local in time estimates. In $H^{1}$ this value is $\sigma=\frac{2}{N-2}$, which is indeed the critical value for which $H^{1} \hookrightarrow L^{\sigma+2}(N>3)$. In $L^{2}$ the critical value is $\sigma=\frac{2}{N-2}$ as well.

Using the same methods than above or in [?], it is possible to prove :

Theorem 4 i) For all $\left(A_{0}, \nu_{0}\right)$ belonging to $H^{s} \times H^{s}$ with $s>N / 2$, equation (??) for the initial data $A(0)=A_{0}, \nu(0)=\nu_{0}$, has a unique solution in $L^{\infty}\left(0, T ; H^{s}\right)$ for a small enough $T$ and solutions depend continuously on the initial data.

ii) For all $\left(A_{0}, \nu_{0}\right)$ belonging to $H^{1} \times H^{1}$ and $\sigma<\frac{2}{N-2}$, equation (??) has a unique solution in $L^{\infty}\left(0, T ; H^{1}\right)$ for a small enough $T$.

iii) For all $\left(A_{0}, \nu_{0}\right)$ belonging to $L^{2} \times L^{2}$ and $\sigma<\frac{2}{N-2}$, equation (??) has a unique solution in $L^{8 / N}\left(0, T ; L^{2(1+\sigma)}\right) \cap \mathcal{C}\left([0, T] ; L^{2}\right)$ for a small enough $T$.

iv) For all $\left(A_{0}, \nu_{0}\right)$ belonging to $H^{2} \times L^{4}$, equation (??) for the initial data $A(0)=$ $A_{0}, \quad \nu(0)=\nu_{0}$, has a unique solution in $\mathcal{C}\left(0, T ; H^{2}\right) \times \mathcal{C}\left(0, T ; L^{4}\right)$ for a small enough $T$ and solutions depend continuously on the initial data.

We will not give any detailed proof of this result since, as we mentioned above, the methods have already been used. The only difference is that estimates are a 
little more awkward.

\section{Perspectives.}

As stressed in the introduction, the main goals to find new results on the Cauchy problem for Schrödinger-Debye equations were first to give a theoretical background for a numerical approach and second to make a step towards the study of the long time behavior of solutions to these equations. This second aim seems difficult to achieve and numerics would be helpful to give us a first guess of what the result may be.

In the context of Theorem ?? i) for example (see [?]), we already know that, as $\tau$ tends to zero, solutions to (??) tend to the solutions to the cubic nonlinear Schrödinger equation

$$
i \frac{\partial}{\partial t} A+\Delta A=\varepsilon|A|^{2} A .
$$

In the case when $\varepsilon$ is positive solutions to (??) exists for all time, whereas in the case when $\varepsilon$ is negative (??) exhibits solutions that blow up in finite time. (Both signs are physical depending on the type of medium we consider). We may expect to find a similar behavior for the Schrödinger-Debye equations. There is no hope to find different results for small $\tau$ and for greater ones since a rescaling of the equations show that the behavior will be the same.

We might think of a Viriel-type identity to prove a finite-time blow-up result. Here we do not dispose of a conservation law but only of a relation like

$$
\frac{d}{d t}\left(\frac{1}{2} \int|\nabla A(t)|^{2} d x+\varepsilon \int|A(t)|^{4} d x-\frac{\tau^{2}}{\varepsilon} \int\left|\frac{\partial \nu}{\partial t}\right|^{2} d x\right)=\frac{2 \tau}{\varepsilon} \int\left|\frac{\partial \nu}{\partial t}\right|^{2} d x
$$

Using this formulation we might think of obtaining a partial result like the one obtained by Merle [?] for the Zakharov equation. All such attempts have been unsuccessful until now. 


\section{References}

[1] B. Bidégaray Etude d'équations intervenant en optique non linéaire. C. R. Acad. Sci., Série I, 319, 361-364 (1994)

[2] B. Bidégaray On the Cauchy problem for systems occurring in nonlinear optics. Adv. Diff. Equ., 3, 473-496 (1998)

[3] B. Bidégaray Crank-Nicolson schemes for Schrödinger-Debye equations. in preparation

[4] T. Kato Nonlinear Schrödinger equations. Lecture Notes in Physics, 345 (1988)

[5] F. Merle Blow-up results of Viriel type for Zakharov equations. Commun. Math. Phys., 175, 433-455 (1996)

[6] T. Ozawa, Y. Tsutsumi Existence and Smoothing Effect of Solutions for the Zakharov Equations. Publ. Res. Inst. Math. Sci., 28, 329-361 (1992)

[7] Y. Shibata, Y. Tsutsumi Local existence of solution for the initial boundary value problem of fully nonlinear wave equation. Nonl. Anal., TMA, 11, 335365 (1987) 